\title{
Risk Attitudes, Buying and Selling Price for a Lottery and Simple Strategies
}

\author{
Michał Lewandowski*
}

Submitted: 29.03.2013, Accepted: 16.05.2013

\begin{abstract}
This paper defines the concept of simple strategy and introduces three kinds of simple strategies: wealth-invariant, scale-invariant and "wealthier-accept more". For three commonly used utility function families: CARA, CRRA and DARA equivalent characterizations are obtained in terms of the corresponding simple strategy, in terms of the buying and selling price properties, and in terms of the utility function properties as expressed by Cauchy functional equations. Moreover, an extension of famous Pratt (1964) theorem is proved which involves buying price for a lottery as an alternative measure of comparative risk aversion. Additionally a number of propositions on both selling and buying price for a lottery and CRRA utility class are proved.
\end{abstract}

Keywords: wealth-invariance, scale-invariance, CARA, CRRA, DARA, risk aversion, buying and selling price for a lottery.

JEL Classification: D81, D03, C91

*Warsaw School of Economics, e-mail: michal.lewandowski@sgh.waw.pl 


\section{Introduction}

The main goal of this paper is to present four alternative characterizations of the three well known classes of risk attitudes: CARA, DARA and CRRA. The four equivalent characterizations are based on the properties of the following functions:

$$
\begin{aligned}
& \text { absolute and relative risk aversion } \\
& \text { buying and selling price for a lottery } \\
& \text { simple strategy }
\end{aligned}
$$$$
\text { Bernoulli utility function }
$$

The first of the aforementioned representations is based on local risk attitudes defined by Pratt (1964) and Arrow (1965). Within this representation three classes of individual risk attitudes and the associated Bernoulli utility functions will be discussed, namely constant absolute risk aversion (CARA), decreasing absolute risk aversion (DARA $)^{1}$ and a subset thereof - constant relative risk aversion (CRRA). The second representation is given by the properties of buying and selling price for a lottery, the concepts defined by Raiffa (1968). Buying price for a lottery is a maximal sure amount which the decision maker is willing to pay to participate in a lottery. Selling price for a lottery is a minimal sure amount which the decision maker is willing to accept to forgo the right to play a lottery. The alternative names which are often used in non-expected utility theories and experimental work are willingness to pay (WTP) for a lottery and willingness to accept (WTA) for a lottery, respectively. Within this representation buying and selling price properties will be considered in separation as well as the way they are linked together. In case of CRRA both the properties of classic buying and selling price for nominal gambles as well as the properties of buying and selling price designed for multiplicative gambles will be analyzed. It will be demonstrated in what sense CARA and buying and selling price for nominal gambles are analogous to CRRA and buying and selling price for multiplicative gambles. Buying and selling price representation might shed light on a recent experimental evidence documenting large spreads between elicited buying and selling prices for the same lottery as well as preference reversal phenomenon. These issues are analyzed in the accompanying paper Lewandowski (2011). The third representation involves the concept of a simple strategy. Simple strategy recommends whether to accept a given gamble or not only on the basis of the gamble itself and the initial wealth which the decision maker is endowed with prior to taking the decision. In a dynamic setting simple strategy corresponds to the notion of a Markov stationary strategy.

Wealth-invariant simple strategy is a strategy which for any gamble does not depend on initial wealth. If the strategy is not wealth-invariant, it is wealth-varying. Among

\footnotetext{
${ }^{1}$ In this paper DARA means strictly decreasing absolute risk aversion.
}

CEJEME 5: 1-34 (2013) 
Risk Attitudes, Buying and Selling...

wealth-varying simple strategies we focus on "wealthier-accept more" simple strategy for which the acceptance set increases when initial wealth increases. A special case of "wealthier-accept more" simple strategy is scale-invariant simple strategy which does not depend on scale. If a gamble is accepted at some wealth level, then if the gamble's outcomes and wealth are multiplied by some positive factor, the new gamble will be accepted at a new wealth level.

Finally, it will be demonstrated that CARA and CRRA class of utility functions can be derived from functional equations which belong to the Cauchy family.

Within Expected Utility framework, for each class of risk attitudes (CARA, DARA, CRRA), the equivalence of four representations (based on measures of risk aversion, buying/selling price, simple strategy and Bernoulli utility function) will be proved. The following diagram illustrates the idea behind these results: The first three

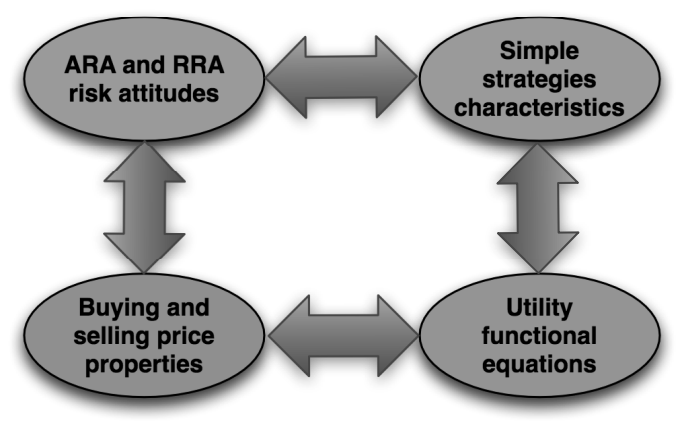

Figure 1: Diagrammatic representation of the equivalency results

representations described above will be dealt with in section 4, whereas the last representation involving Cauchy functional equations will be analyzed in section 5 . Even though some parts of these results are well known it was decided to put them all together both for the sake of completeness as for the sake of their novel formulation and the unifying method of proof. For example, the equivalence between CARA, wealth invariance and constant buying and selling price has been around since Pratt (1964), however the way it's stated in this paper is much more straightforward and ready to use. The concept of simple strategy is a novel component introduced by Foster and Hart (2007) in the context of constant relative risk aversion class ${ }^{2}$. "Wealthier-accept more" and wealth-invariant simple strategies are defined for the purpose of this paper. The result, which is often forgotten among economists is the one for constant relative risk aversion utility class. For example, in section 4 Proposition 1 Barbeis and Huang (2009) restrict certainty equivalent functional, which they denote $\mu(\cdot)$, to the case of constant relative risk aversion utility functions. They claim that "the same method

\footnotetext{
${ }^{2}$ Foster and Hart (2007) introduced the concept of homogeneous simple strategy.
} 
Michał Lewandowski

of proof used in Proposition 1 can also be applied to other explicitly defined forms of $\mu(\cdot)$, whether expected utility or not that satisfy the homogeneity property ${ }^{3 "}$. In the paper it is shown that except for CRRA there are no other expected utility forms of certainty equivalent that satisfy homogeneity property and hence the statement Barbeis and Huang (2009) make is not correct (or at least not precise). The result concerning CRRA as well as other characterization results in this paper may help in clarifying some of the imprecise statements from the literature such as the one cited above.

The three characterization results in this paper show that the same message can be delivered in four different ways depending on the needs and on the context. For example to assume CRRA is equivalent to assume positive homogeneity of buying and selling prices, and also equivalent to assuming scale-invariance of simple strategy. Moreover, CRRA utility function satisfies a simple functional equation. The formulation in terms of simple strategies makes the notion of CRRA more intuitive since it is expressed directly in terms of the decision maker's actions. Therefore, when testing the hypothesis of CRRA, it might be useful to test instead whether simple strategy is homogeneous. Alternatively, if the experimenter has access to data on buying and selling price, it might be more straightforward to test homogeneity of these. Thus the characterization results form the bridge between different formulations.

Another result of this paper shows that buying price for a lottery can be used to compare risk aversion of two agents in an equivalent way as selling price for a lottery and other methods laid out in Pratt (1964) famous theorem ${ }^{4}$. This result is an extension of Pratt (1964) theorem which characterizes comparative risk aversion. It might be useful in testing comparative risk aversion when the data on individual buying prices is available whereas the data on individual selling prices is not. Also, using buying price has one technical advantage over using selling price.

Buying price exhibits the so called delta property ${ }^{5}$ whereas selling price in general does not ${ }^{6}$. It means that calibration process in case of buying price might be much easier than in case of selling price.

The paper is divided into three parts. In section 2 the main definitions and assumptions of the model are introduced in a formal way. In section 3 the idea of nominal and multiplicative gambles and the way risk aversion is incorporated for these two concepts is presented. In section 4 four main results are stated. In section 5 additional results are presented which introduce Cauchy functional equations and their equivalence with CRRA and CARA utility class. Moreover, certain theoretical properties of buying and selling price are stated. In section 7 main results are proved together with a number of auxiliary lemmas and propositions.

\footnotetext{
${ }^{3}$ I.e. positive homogeneity of certainty equivalence.

${ }^{4}$ Selling price for a lottery is the negative or risk compensation used in Pratt (1964).

${ }^{5}$ For details see appendix lemma 15.

${ }^{6}$ The exception is CARA class for which selling price exhibits delta property.
}

CEJEME 5: 1-34 (2013) 
Risk Attitudes, Buying and Selling...

\section{The model}

In this section the assumptions and definitions of buying and selling price and the notion of simple strategies are introduced.

Assumption 1. Preferences obey expected utility axioms. Bernoulli utility function $U: \mathbb{R} \rightarrow \mathbb{R}$ is twice continuously differentiable, strictly increasing and strictly concave.

Definition 1. A lottery $\mathbf{x}$ is a real- and finite-valued random variable with finite support. A maximal loss of lottery $\mathbf{x}$ is defined as $\min (\mathbf{x})=\min \operatorname{supp}(\mathbf{x})$. Wealth $W$ is a real number.

Although most of the results that follow are true for more general lotteries, the finite support assumption is adopted for the sake of simplicity. Now buying and selling price for a lottery given wealth level are defined along the lines of Raiffa (1968). To avoid repetitions, statements of the form: "Given utility function $U$ satisfying assumption 1, any lottery $\mathbf{x}$ and wealth $W \ldots$... will henceforth be skipped.

Definition 2. Define selling price $S(W, \mathbf{x})$ and buying price $B(W, \mathbf{x})$ for a lottery $\mathbf{x}$ at wealth $W$ as follows:

$$
\begin{aligned}
& \mathrm{E} U[W+\mathbf{x}]=U[W+S(W, \mathbf{x})] \\
& \mathrm{E} U[W+\mathbf{x}-B(W, \mathbf{x})]=U(W)
\end{aligned}
$$

The domain of $S$ and $B$, i.e. all admissible pairs $(W, \mathbf{x})$, is assumed to be such that the left-hand side and the right-hand side of the above equations are well defined, given the domain of $U^{7}$. The space of such admissible pairs will be denoted by $\mathcal{X}$. Notice that functions $B$ and $S$ are then well defined by assumption 1 .

Definition 3. An individual's simple strategy $s: \mathcal{X} \rightarrow\{1,0\}$ assigns to each admissible pair $(W, \mathbf{x})$ either value 1 or 0 , representing "Accept $\mathbf{x}$ at $W$ " or "Reject $\mathbf{x}$ at $W^{\prime \prime}$, respectively.

Since the aim in this paper is to link the concept of simple strategy to expected utility maximization, the following non-triviality assumption is imposed:

Assumption 2. For any non-degenerate lottery $\mathbf{x}$ and wealth $W>0$, there exists a unique $p_{W, \mathbf{x}}^{*} \in(\min (\mathbf{x}), \mathrm{E}[\mathbf{x}])$ such that:

$$
\begin{aligned}
& s(W, \mathbf{x}-p)=1, p \leq p_{W, \mathbf{x}}^{*} \\
& s(W, \mathbf{x}-p)=0, p>p_{W, \mathbf{x}}^{*}
\end{aligned}
$$

\footnotetext{
${ }^{7}$ If the utility function is not defined over the whole real line as is the case of CRRA utility function, one has to make sure first that both sides of the above equations are well defined and second that the equality has a solution. For details see Lewandowski (2011).
} 
This assumption asserts that there are other lotteries which are accepted beyond those with no losses and there are other lotteries which are rejected beyond those with negative expectation. This assumption imposes monotonicity of preferences and risk aversion. Notice that in expected utility setup given wealth $W$ and lottery $\mathbf{x}$, $p_{W, \mathbf{x}}^{*}=B(W, \mathbf{x})$.

Definition 4. Simple strategy is wealth-invariant if

$$
s\left(W_{1}, \mathbf{x}\right)=1 \Longleftrightarrow s\left(W_{2}, \mathbf{x}\right)=1, \forall W_{1}, W_{2}
$$

And the above holds for all $\mathbf{x}$ that are accepted.

It follows that if simple strategy is wealth-varying (i.e. not wealth-invariant) then there exists lottery $\mathbf{x}$ and two different wealth levels $W_{1}, W_{2}$ such that:

$$
s\left(W_{1}, \mathbf{x}\right)=1 \wedge s\left(W_{2}, \mathbf{x}\right)=0
$$

To understand the difference between wealth-varying and wealth-invariant simple strategy suppose there is a lottery with only positive outcomes. Any individual who prefers less to more will accept such lottery irrespective of initial wealth level. It does not mean however that the underlying simple strategy is wealth-invariant. That is the reason why wealth-varying simple strategy is defined using the existence quantifier and not the universal quantifier.

Two kinds wealth-varying simple strategies are introduced:

Definition 5. Wealth-varying simple strategy is of "wealthier-accept more" type if

$$
s\left(W_{1}, \mathbf{x}\right)=1 \wedge s\left(W_{2}, \mathbf{x}\right)=0 \Rightarrow W_{1}>W_{2}
$$

Definition 6. Wealth-varying simple strategy is scale-invariant or homogeneous if

$$
s(W, \mathbf{x})=1 \Longleftrightarrow s(\lambda W, \lambda \mathbf{x})=1, \forall W, \forall \lambda>0
$$

And this holds for all $\mathbf{x}$ that are accepted.

The goal here is to analyze risk attitudes. It is convenient to define two kinds of utility function transformations which do not alter the underlying risk attitudes.

Lemma 1. If $U$ is a utility function, relative and absolute risk aversion function is unique up to the following transformation of $U$ :

$$
\mathcal{A}:\{v(x)=a U(\delta x)+b\}
$$

where $\delta=\{1,-1\}, a, b \in \mathbb{R}$ and $a>0$.

Furthermore, for $a<0$, relative and absolute risk aversion changes only its sign.

M. Lewandowski

CEJEME 5: 1-34 (2013) 
Risk Attitudes, Buying and Selling...

Proof. Since Bernoulli utility function is unique only up to affine transformation $a u(\cdot)+b, A>0$ represents the same risk attitudes as $u(\cdot)$. Furthermore, relative and absolute risk aversion obtained from $u(x)$ and $u(-x)$ is the same in sign and magnitude and that from $-u(x)$ and $-u(-x)$ is of opposite sign but the same magnitude.

Observe that if $u(x)$ is increasing and concave, then $u(-x)$ is decreasing and concave, $-u(x)$ is decreasing and convex, and $-u(-x)$ is increasing and convex. The absolute value of Arrow, Pratt risk aversion measures is however the same for all these functions. The use of such transformations will prove useful when characterizing different classes of risk attitudes by means of Cauchy family functional equations.

\section{Nominal and multiplicative gambles}

\subsection{Nominal gambles and wealth invariance vs multiplicative gambles and scale invariance}

Suppose an individual with wealth $W$ faces a choice whether to accept or reject gamble $\mathbf{x}$. The consequences of $\mathbf{x}$ are monetary. Consider two different objectives this individual might have:

a. wealth from accepting $\mathbf{x}$ should increase on average in nominal terms

b. return from $\mathbf{x}$ should be positive on average

Let's define random return from $\mathbf{x}$ given wealth $W>0$ as $\mathbf{h}=1+\frac{\mathbf{x}}{W}$. Gamble $\mathbf{x}$ is called a nominal gamble since its units are expressed in nominal terms. Gamble $\mathbf{h}$ is called a multiplicative gamble and it is dimensionless. It is assumed that the maximal loss of $\mathbf{x}$ is strictly smaller than $W$ so that gamble $\mathbf{h}$ takes only positive values. Aumann and Serrano (2008) suggest that financial instruments may be regarded as such multiplicative gambles. Notice that nominal gamble does not depend on wealth and the acceptance of such gamble using the first of the above criteria does not depend on initial wealth $W$. On the other hand multiplicative gamble does depend on initial wealth and the acceptance of such gamble using the second of the above criteria also depends on initial wealth. However, what the multiplicative gamble is invariant to is scale. No matter in what units consequences ${ }^{8}$ are measured, or, alternatively, whether both initial wealth and the nominal gamble $\mathbf{x}$ is multiplied by the same positive factor, the resulting multiplicative gamble remains unchanged.

Notice that the two criteria above do not invoke any arguments on risk aversion. In fact, the first criterion amounts to risk neutrality in a classical sense. Similarly, it is useful to think of the second criterion as risk neutrality for multiplicative gambles. It will be shown below that the two widely used classes of utility functions, CARA and CRRA, are generalizations of the above two criteria, respectively - generalizations

\footnotetext{
${ }^{8}$ Both, values of $\mathbf{x}$ and initial wealth $W$.
} 
in the sense of introducing risk aversion, specific to nominal gambles and wealth invariance in the first case and specific to multiplicative gambles and scale invariance in the second case.

First, notice that the first criterion above is equivalent to evaluating the arithmetic mean of a nominal gamble:

$$
\text { accept } \mathbf{x} \Longleftrightarrow \mathrm{E}_{\mathrm{a}}(\mathrm{W}+\mathbf{x}) \geq \mathrm{W} \Longleftrightarrow \mathrm{E}_{\mathrm{a}}(\mathbf{x}) \geq 0
$$

where $\mathrm{E}_{\mathrm{a}}$ denotes arithmetic mean operator. ${ }^{9}$ The second criterion, on the other hand, is equivalent to the following:

$$
\text { accept } \mathbf{h} \Longleftrightarrow W \times \mathrm{E}_{\mathrm{g}}(\mathbf{h}) \geq \mathrm{W} \Longleftrightarrow \mathrm{E}_{\mathrm{g}}(\mathbf{h}) \geq 1
$$

where $\mathrm{E}_{\mathrm{g}}$ denotes geometric mean operator. The detailed explanation why the above is true may be found in the appendix at the end of this paper.

Since $\mathbf{h}$ takes only positive values, the condition on the right of (7) may be rewritten as:

$$
\begin{aligned}
\log \mathrm{E}_{\mathrm{g}}(\mathbf{h}) & \geq 0 \\
\mathrm{E}_{\mathrm{a}} \log (\mathbf{h}) & \geq 0 \\
\mathrm{E}_{\mathrm{a}} \log \left(1+\frac{\mathbf{x}}{W}\right) & \geq 0 \\
\mathrm{E}_{\mathrm{a}} \log (W+\mathbf{x}) & \geq \log W
\end{aligned}
$$

The conclusion from this analysis is the following: the second criterion stating that the return from $\mathbf{x}$ should be positive on average is equivalent to expected utility from accepting $\mathbf{x}$ is at least as high as from rejecting $\mathbf{x}$, where the Bernoulli utility function is logarithmic. So a kind of risk neutrality for multiplicative gambles (the second criterion) is equivalent to logarithmic risk aversion in classical sense i.e. for nominal gambles.

This fact is a basis for Foster and Hart (2007) paper. It will be demonstrated now that the same transformation of the two criteria a. and b. discussed in this section lead to CARA and CRRA class, respectively. The goal is to introduce risk aversion into criteria a. and b. As noted before, criteria a. and b. are equivalent to the following two conditions, respectively:

$$
\begin{aligned}
\mathrm{E}_{\mathrm{a}} \mathbf{x} & \geq 0 \\
\mathrm{E}_{\mathrm{a}} \log (\mathbf{h}) & \geq 0
\end{aligned}
$$

Consider, CARA utility function $U(x)=\frac{1-e^{-\beta x}}{\beta}$. By definition, exchanging $x$ with $U(x)$ in the first of the above equation gives rise to expected utility decision making

\footnotetext{
${ }^{9}$ Generally, in the whole thesis, if not explicitly stated otherwise E denotes arithmetic mean operator. Here, different notation is used to stress the difference to geometric mean operator $\mathrm{E}_{\mathrm{g}}$, which will also be used.
}

M. Lewandowski

CEJEME 5: 1-34 (2013) 
Risk Attitudes, Buying and Selling...

with CARA Bernoulli utility function. Now consider exchanging $\log x$ with $U(\log x)$ in the second case:

$$
U(\log x)=\frac{1-e^{-\beta \log x}}{\beta}=\frac{1-x^{-\beta}}{\beta}
$$

Now let's define $\beta=-(1-\alpha)$ to obtain:

$$
U(\log x)=\frac{x^{1-\alpha}-1}{1-\alpha}
$$

And hence equation (8) will change to:

$$
\mathrm{E}_{\mathrm{a}}\left[\frac{\mathbf{h}^{1-\alpha}-1}{1-\alpha}\right] \geq 0
$$

And this is expected utility decision making with CRRA Bernoulli utility function.

\subsection{Buying and selling price for multiplicative gambles}

In the previous section, the concepts of buying and selling price for a lottery were introduced. These concepts were specifically designed to deal with nominal gambles. It is possible to define similar concepts for multiplicative gambles. If $\mathbf{x}$ is a nominal gamble and $W>L(\mathbf{x})$ is initial wealth, denote $\mathbf{h}$ as multiplicative gamble and write $\mathbf{h}=\frac{W+\mathbf{x}}{W}$.

Definition 7. Given utility function: $U: R^{+} \rightarrow R$, a multiplicative gamble $\mathbf{h}$, selling return price $s(W, \mathbf{h})$ and buying return price $b(W, \mathbf{h})$ for a multiplicative gamble $\mathbf{h}$ at wealth $W$ are defined as follows:

$$
\begin{aligned}
& \mathrm{E} U[W \mathbf{h}]=U[W s(W, \mathbf{h})] \\
& \mathrm{E} U\left[\frac{W \mathbf{h}}{b(W, \mathbf{h})}\right]=U(W)
\end{aligned}
$$

The interpretation of these two measures is the following. Selling return price $s(W, \mathbf{h})$ is the minimal sure return which an individual whose preferences are represented by $U$ would demand to forgo random return $\mathbf{h}$. On the other hand, buying return price $b(W, \mathbf{h})$ is the maximal sure return which an individual is willing to forgo for the right to play gamble $\mathbf{h}$. It is easy to show in as similar way to that in lemma 4 that for non-degenerate $\mathbf{h}$, both $b(W, \mathbf{h})$ and $s(W, \mathbf{h})$ lie in the interval $\left(1-\frac{L(\mathbf{x})}{W}, 1+\frac{\mathrm{E}[\mathbf{x}]}{W}\right)$. Since it is assumed that $W>L(\mathbf{x})$, it is guaranteed that all the arguments in the above two equations are non-negative and hence by monotonicity and continuity of $U$, the two equations are well defined and there exist unique selling and buying return prices. There is a simple relationship between selling price for nominal lottery $\mathbf{x}$ and selling return price for multiplicative gamble:

$$
s(W, \mathbf{h})=1+\frac{S(W, \mathbf{x})}{W}
$$


An analogous relationship between $b(W, \mathbf{h})$ and $B(W, \mathbf{x})$ is however more complex and, in general, can only be given in an implicit form:

$$
\mathrm{E} U\left(\frac{W \mathbf{h}}{b(W, \mathbf{h})}\right)=\mathrm{E} U(W \mathbf{h}-B(W, \mathbf{x}))
$$

\section{Main equivalence results}

The first result below is essentially just a reformulation and combination of results appearing in Pratt (1964) and Raiffa (1968). They are restated in a convenient form

for completeness. The proofs of all the following results, including the first one are provided in section 7 .

Proposition 4.1 (CARA). The following three statements are equivalent:

i. strategy is wealth-invariant

ii. Bernoulli utility function exhibits CARA

iii. buying and selling price are independent from wealth and equal i.e.

$$
B(W, \mathbf{x})=S(W, \mathbf{x})=C_{\alpha}, \forall W
$$

where $\alpha$ is absolute risk aversion coefficient and $C_{\alpha}$ takes real values and depends only on $\alpha$.

Proposition 4.2 (DARA). The following three statements are equivalent:

i. strategy is wealthier - accept more

ii. Bernoulli utility function exhibits DARA

iii. buying and selling price are increasing in $W$ and

$$
B(W, \mathbf{x})>0 \Longleftrightarrow B(W, \mathbf{x})<S(W, \mathbf{x})
$$

Proposition 4.3 (CRRA). The following four statements are equivalent:

i. strategy is scale-invariant

ii. Bernoulli utility function exhibits CRRA

iii. buying and selling price for any lottery are homogeneous of degree one i.e.

$$
\begin{aligned}
& S(\lambda W, \lambda \mathbf{x})=\lambda S(W, \mathbf{x}), \forall \lambda>0 \\
& B(\lambda W, \lambda \mathbf{x})=\lambda B(W, \mathbf{x}), \forall \lambda>0
\end{aligned}
$$

M. Lewandowski

CEJEME 5: 1-34 (2013) 
Risk Attitudes, Buying and Selling...

$i v$. buying and selling return prices for any multiplicative lottery are independent from wealth and equal i.e.

$$
b(W, \mathbf{h})=s(W, \mathbf{h})=C_{\beta}, \forall W
$$

where $\beta$ is relative risk aversion coefficient and $C_{\beta}$ takes real values and depends only on $\beta$. Additionally,

$$
\begin{aligned}
& b(W, \lambda \mathbf{h})=\lambda b(W, \mathbf{h}), \forall \lambda>0 \\
& s(W, \lambda \mathbf{h})=\lambda s(W, \mathbf{h}), \forall \lambda>0
\end{aligned}
$$

In the last proposition concerning CRRA class of utility function, an additional item has been added which characterizes buying and selling return prices. As suggested by Roberto Serrano, it is useful to see that buying and selling return price in case of CRRA share the same characteristics with buying and selling price in case of CARA. In particular, conditions (12) and (15) make it clear that in case of CRRA buying and selling return price are equal to each other and independent from wealth the same way as in case of CARA buying and selling price are equal to each other and independent from wealth. Conditions (16) and (17) are on the other hand specific for buying and selling return prices in case of CRRA. These conditions state that buying and selling return prices are both homogeneous in a gamble. Since buying and selling return prices were designed to deal with multiplicative gambles and CRRA class is a scale-invariant, these conditions are perhaps more intuitive then the analogous conditions (13) and (14) which concern buying and selling price, the concepts designed for nominal gambles.

The above three propositions characterize three widely used risk attitude classes of utility function. Certain parts of these propositions are already known in the literature and some are novel. The advantage lies in putting all these results together and offering a unifying way to prove them. In applied work these results should be especially useful since they allow to interchangeably use the notions of risk attitude classes of utility function represented by absolute and relative risk aversion, the corresponding simple strategies defined above and properties of buying and selling price for a lottery. It should help in testing of risk attitudes, as it might be simpler to test either buying and selling price for a lottery or simple strategies depending on the context. Experiments should be designed as naturally as possible. Subjects are reluctant to engage in considerations regarding abstract notions. Here, the advantage of a simple strategy notion is apparent due to its direct reference to actions. In other contexts on the other hand, where trading atmosphere is to be created in experimental settings, buying and selling price for a lottery might be more appropriate. The three equivalent characterizations of risk attitudes classes of utility function should be of advantage both in theoretical as well as applied work. Notice further that a number of useful observation might be made after careful examination of the above results. For example, since CRRA is a subclass of DARA, it is therefore the case that 
homogeneous simple strategy is a subset of "wealthier-accept more" simple strategies. Such conclusion is not obvious without the propositions above.

The next result that follows is an extension of Pratt (1964) famous result on comparative risk aversion. It establishes an equivalence between buying and selling price as an index for greater-risk aversion relation.

Proposition 4.4. For two different utility functions $U_{1}(\cdot)$ and $U_{2}(\cdot)$ with nonincreasing absolute risk aversion, let $S_{1}(W, \mathbf{x}), B_{1}(W, \mathbf{x})$ and $S_{2}(W, \mathbf{x}), B_{2}(W, \mathbf{x})$ be the corresponding buying and selling price functions. The following equivalence holds:

$$
\begin{gathered}
\forall W \forall \mathbf{x}: \exists \delta>0\left|x_{i}\right|<\delta \forall i \in\{1, \ldots, n\} \\
S_{1}(W, \mathbf{x})>S_{2}(W, \mathbf{x}) \Longleftrightarrow B_{1}(W, \mathbf{x})>B_{2}(W, \mathbf{x})
\end{gathered}
$$

This proposition may be useful as well in both theoretical and empirical work. Since buying price for a lottery exhibits delta property no matter what the risk attitude, and selling price in general does not ${ }^{10}$ it might be simpler to use buying price as an index of comparative risk aversion since the proposition says that one can use the two indices interchangeably. In empirical settings, one might have data only on buying price for a lottery and not on selling price. In this case inferences regarding selling price for a lottery and hence absolute risk aversion are still possible due to the above result.

\section{Additional results}

\subsection{Additional characterization results}

The following results give further insights into the nature of widely used risk attitudes classes. By means of a couple of simple functional equations one can give alternative proofs to the first equivalence results (i. $\Longleftrightarrow$ ii.) in propositions $4.1,4.2$ and 4.3. The equations which will be analyzed below belong to a Cauchy family of functional equations:
a. $v(x+y)=v(x)+v(y)$
b. $v(x+y)=v(x) v(y)$
c. $v(x y)=v(x)+v(y)$
d. $v(x y)=v(x) v(y)$

It is useful to treat the above functions as transformations described in lemma 1 of the corresponding utility functions. It proves much easier to work with transformations and not directly with utility functions since these transformations are chosen to satisfy

\footnotetext{
${ }^{10}$ Selling price exhibits delta property only in case of CARA.
} 
Risk Attitudes, Buying and Selling...

the simplest functional equation with a given concavity/convexity properties. By lemma 1 it is possible to transform function $v$ into a utility function $U$ which satisfies any desired properties - e.g. normalized conveniently, increasing and concave without changing the risk attitudes properties.

It will be shown that the above four functional equations are equivalent characterizations of risk neutral, CARA, logarithmic and CRRA preferences respectively ${ }^{11}$.

Proposition 5.1. All twice continuously differentiable functions $v: \mathbb{R} \rightarrow \mathbb{R}$ that satisfy the following functional equation: $v(x+y)=v(x)+v(y)$ for all $x, y$ belonging to the domain of $v$, are of the following form:

$$
v(x)=c x, \quad c \in \mathbb{R}
$$

Proof. For $x=y=0, v(0)=v(0)+v(0)$. It implies that $v(0)=0$. Rearrange the equation and divide by $y$ :

$$
\frac{v(x+y)-v(x)}{y}=\frac{v(y)}{y}
$$

Letting $y$ tend to zero and using Hospital rule on the right hand side, the following is obtained:

$$
v^{\prime}(x)=\frac{v^{\prime}(0)}{1}
$$

Define $c=v^{\prime}(0)$ and integrate both sides from 0 to $x$ of the above equation to obtain $v(x)=c x$ as required.

Proposition 5.2. All twice continuously differentiable functions $v: \mathbb{R} \rightarrow \mathbb{R}$ that satisfy the following functional equation: $v(x+y)=v(x) v(y)$ for all $x, y$ belonging to the domain of $v$, are of the following form:

$$
v(x)=e^{c x}, \quad c \in \mathbb{R}
$$

Proof. For $y=0, v(x)=v(x) v(0)$. It implies that $v(0)=1$. Using the equation, it can be written:

$$
\frac{v(x+y)-v(x)}{y}=v(x) \frac{v(y)-1}{y}
$$

Now let $y$ on both sides go to zero. Using Hospital rule on the right hand side it is obtained:

$$
v^{\prime}(x)=v(x) \frac{v^{\prime}(0)}{1}
$$

\footnotetext{
${ }^{11}$ The proofs which are given below rely on the assumption of differentiability of the underlying functions $v$. It is important to stress that differentiablity is not necessary to get the same result. Cauchy proved it in 1821 for continuous functions and Darboux proved it in 1875 for functions which are continuous only at one point. Here, the aim is not to be so general: since risk attitudes described by local measures of risk aversion are the main aim of this paper, the twice continuous differentiability is assumed anyway.
} 
Define $c=v^{\prime}(0)$ and rearrange to obtain:

$$
[\log v(x)]^{\prime}=c
$$

Now integrate both sides from 0 to $x$ and exponentiate on both sides to obtain:

$$
v(x)=e^{c x}
$$

Proposition 5.3. All twice continuously differentiable functions $v: \mathbb{R}^{++} \rightarrow \mathbb{R}$ that satisfy the following functional equation: $v(x y)=v(x)+v(y)$ for all $x, y$ belonging to the domain of $v$, are of the following form:

$$
v(x)=c \log x, \quad c \in \mathbb{R}
$$

Proof. For $y=1, v(x)=v(x)+v(1)$. It implies that $v(1)=0$. let's define $y=1+h$. Now using the equation it can be written:

$$
\frac{v(x(1+h))-v(x)}{x h}=\frac{1}{x} \frac{v(1+h)}{h}
$$

Now let $h$ tend to zero on both sides and apply Hospital rule on the right hand side:

$$
v^{\prime}(x)=\frac{1}{x} \frac{v^{\prime}(1)}{1}
$$

Now define $c=v^{\prime}(1)$ and integrate both sides from 1 to $x$ to obtain:

$$
v(x)=c \log x
$$

Proposition 5.4. All twice continuously differentiable functions $v: \mathbb{R}^{++} \rightarrow \mathbb{R}$ that satisfy the following functional equation: $v(x y)=v(x) v(y)$ for all $x, y$ belonging to the domain of $v$, are of the following form:

$$
v(x)=x^{c}, \quad c \in \mathbb{R}
$$

Proof. For $y=1, v(x)=v(x) v(1)$. It implies that $v(1)=1$. Let's define $y=1+h$. Now using the equation it can be written:

$$
\frac{v(x(1+h))-v(x)}{x h}=\frac{v(x)}{x} \frac{v(1+h)-1}{h}
$$

Now let $h$ tend to zero on both sides and apply Hospital rule on the right hand side:

$$
v^{\prime}(x)=\frac{v(x)}{x} \frac{v^{\prime}(1)}{1}
$$

Now define $c=v^{\prime}(1)$ and rearrange to obtain:

$$
[\log v(x)]^{\prime}=c \frac{1}{x}
$$

Integrate both sides from 1 to $x$ and rearrange to obtain:

$$
v(x)=x^{c}
$$

M. Lewandowski

CEJEME 5: 1-34 (2013) 
Risk Attitudes, Buying and Selling...

It is worth noting that the above propositions could be stated in a stronger form. To prove that any of the four functional equations implies the corresponding function, one does not need to assume that $v$ is twice continuously differentiable. It is true for any continuous or monotonic functions. Furthermore, it requires a full proof only for the first of the four functional equations, as the others may be reduced to it by using appropriate transformation of $v$. This is also the reason why all the four equations belong to one family of Cauchy equations. Suppose function $v$ satisfies $v(x+y)=v(x) v(y)$. Define a transformation of $\mathrm{v}$, namely $g(x)=\log v(x)$. It is straightforward to see that $g(x)$ satisfies $g(x+y)=g(x)+g(y)$, so it has to be that $g(x)=c x$ and going back to the original function $v(x)=e^{c x}$. Similarly, the corresponding transformation of $v$ which satisfies $v(x y)=v(x)+v(y)$ is $g(x)=v\left(e^{x}\right)$ and the corresponding transformation of $v$ which satisfies $v(x y)=v(x) v(y)$ is $g(x)=\log v\left(e^{x}\right)$.

To see how the above functional equations connect to expected utility decision making, suppose that $W$ is initial wealth, $\mathbf{x}$ is a lottery to be chosen and $U$ is a utility function which is transformed from the corresponding function $v$ satisfying one of the four functional equations. Define the following expression $a(W, \mathbf{x})=\mathrm{E} U(W+\mathbf{x})-U(W)$. The list below corresponds to the four functional equations above:

a. $a(W, \mathbf{x})=\mathrm{E} U(\mathbf{x})$

b. $a(W, \mathbf{x})=U(W)(\mathrm{E} U(\mathbf{x})-1)$

c. $a(\lambda W, \lambda \mathbf{x})=\mathrm{E} U(W+\mathbf{x})-U(W)$

d. $a(\lambda W, \lambda \mathbf{x})=U(\lambda)[\mathrm{E} U(W+\mathbf{x})-U(W)]$

The conclusions are the following. In the first case, corresponding to linear utility function, the utility from accepting lottery $\mathbf{x}$ does not depend on $W$. In the second case, corresponding to CARA (without linear) utility function, the acceptance of $\mathbf{x}$ does not depend on $W$ but the utility value from accepting $\mathbf{x}$ depends on $W$. In case c., corresponding to logarithmic utility function, the utility from accepting $\lambda \mathbf{x}$ at $\lambda W$ does not depend on scale $\lambda$. In case d., corresponding to CRRA (without log) utility function, the acceptance of $\lambda \mathbf{x}$ at $\lambda W$ does not depend on scale $\lambda$ but the utility value from accepting it does.

It can therefore be proposed to call linear utility function - totally wealth invariant, logarithmic utility function- totally scale invariant, other than linear CARA functions - acceptance wealth invariant, and other than logarithmic CRRA functions acceptance scale invariant.

The characterization of CRRA utility function may be supplemented by the following lemma:

Lemma 2. Given twice continuously differentiable utility function $u: \mathbb{R}^{++} \rightarrow \mathbb{R}$ and $\theta, \alpha \in \mathbb{R}, \theta>0, \alpha \neq 0$, the following holds:

$$
u(\theta x)=\theta^{\alpha} u(x) \Longleftrightarrow u(x)=A x^{\alpha}
$$


where $A>0$ is a constant.

Proof. Sufficiency is straightforward. Let's prove necessity. Suppose $\theta=1+\frac{h}{x}$. Then

$$
\lim _{h \rightarrow 0} \frac{\left(1+\frac{h}{x}\right)^{\alpha}-1}{h} u(x)=u^{\prime}(x)
$$

Using Hospital's rule

$$
\lim _{h \rightarrow 0} \frac{\alpha\left(1+\frac{h}{x}\right)^{\alpha-1} \frac{1}{x}}{1} u(x)=u^{\prime}(x)
$$

And this is equivalent to:

$$
\frac{u^{\prime}(x)}{u(x)}=\frac{\alpha}{x}
$$

Define $u(1)=A$, then

$$
\int_{1}^{x}[\log u(t)]^{\prime} d t=\alpha \int_{1}^{x}[\log t]^{\prime} d t
$$

Hence

$$
\log \frac{u(x)}{A}=\alpha \log x
$$

And finally

$$
u(x)=A x^{\alpha}
$$

Notice that since Bernoulli utility function is unique up to affine transformation, utility function of the form $u(x)=A x^{\alpha}$, for $A>0$ and $\alpha \neq 0$ is equivalent to utility function of the form $u(x)=a x^{\alpha}+b$, where $a>0$ and $b \in \mathbb{R}$. Hence for any CRRA utility function ${ }^{12}$ there exists an equivalent utility function that is homogeneous of some degree different than zero.

\subsection{Additional results on buying and selling price}

This paper is part of the research project developing a theory of buying and selling price for a lottery. Therefore, apart from results which may be useful for their own sake, the following are some additional results describing properties of buying and selling price. In particular it turns out that buying and selling price for a lottery $\mathbf{x}$ are concave in $W$ for CRRA utility functions.

Proposition 5.5. For any wealth $W$ and any non-degenerate lottery $\mathbf{x}$, such that $B(W, \mathbf{x})$ and $S(W, \mathbf{x})$ are well defined, the following holds for CRRA utility function:

$$
B(W, \mathbf{x}) \text { and } S(W, \mathbf{x}) \text { are strictly concave in } W
$$
it.

${ }^{12}$ Except for the logarithm, but since it is a limiting case of a power utility function one can ignore

CEJEME 5: 1-34 (2013) 
Risk Attitudes, Buying and Selling...

$$
\begin{aligned}
& B(\theta W, \mathbf{x})+B((1-\theta) W, \mathbf{y})<B(W, \mathbf{x}+\mathbf{y}), \forall \theta \in(0,1) \\
& S(\theta W, \mathbf{x})+S((1-\theta) W, \mathbf{y})<S(W, \mathbf{x}+\mathbf{y}), \forall \theta \in(0,1)
\end{aligned}
$$

Proof. In section 7 .

Below the additivity properties of buying and selling price are examined. It turns out that buying price is sub-additive for all strictly increasing and strictly concave utility functions and selling price is sub-additive only for CRRA subclass of such utility functions.

Proposition 5.6. Suppose lottery $\mathbf{x}$ has at least two values in the support. Let $U$ be a strictly increasing and strictly concave function. For any $W$ and any lottery $\mathbf{x}$ such that buying and selling price are well defined and $n \in \mathbb{Z}, n>1$, the following holds:

$$
B(W, n \mathbf{x})<n B(W, \mathbf{x})
$$

Proof. In section 7 .

For buying price the result holds for all concave functions ${ }^{13}$. For selling price an equivalent result does not hold in general for all concave functions. To see this consider the following utility function:

$$
U(x)= \begin{cases}2 x & \text { for } x<1 \\ \frac{1}{2} x+\frac{3}{2} & \text { for } x \geq 1\end{cases}
$$

This is clearly a continuous weakly concave function. Now consider the following lottery: $\mathbf{x}=\left(5, \frac{1}{2} ; 0, \frac{1}{2}\right)$, i.e. a lottery which gives 5 or 0 with equal probabilities. Simple calculation delivers that the selling price ${ }^{14}$ for this lottery is equal to 1 . Now, let's consider another lottery $\mathbf{y}=2 \mathbf{x}=\left(10, \frac{1}{2} ; 0, \frac{1}{2}\right)$. Selling price for this lottery is equal to $\frac{7}{2}$. Hence, we have $S(0,2 \mathbf{x})=\frac{7}{2}>2=2 S(0, \mathbf{x})$.

It is clear therefore that the result equivalent to proposition 5.6 for selling price does not hold in general. However it does hold for certain classes of utility functions. Below it is shown that it holds for the CRRA class:

Proposition 5.7. Suppose lottery $\mathbf{x}$ has at least two values in the support. Let $U$ be a strictly increasing and strictly concave CRRA function. For any $W$ and any lottery $\mathbf{x}$ such that buying and selling price are well defined and $n \in \mathrm{Z}, n>1$, the following holds:

$$
S(W, n \mathbf{x})<n S(W, \mathbf{x})
$$

Proof. In section 7.

\footnotetext{
${ }^{13}$ Even if the function is not strictly concave, the result is still true if strict inequality is replaced by the weak inequality in equation (18)above.

${ }^{14}$ Without loss of generality consider $W=0$ is considered.
} 
The above results may be used to analyze selling and buying price for several lotteries with given dependence structure. For illustration, suppose utility function is CRRA and let's take a sequence of $n>1$ identically distributed lotteries $\mathbf{x}_{\mathbf{i}}=(-x, 1 / 2 ; x, 1 / 2)$, where $x>0$ and $i \in\{1,2, \ldots, n\}$. The goal is to find a buying and selling price for a sum of such lotteries: $\mathbf{y}=\sum_{i=1}^{n} \mathbf{x}_{\mathbf{i}}$. Such a sum is a new lottery which is not identified until the joint distribution between lotteries $\mathbf{x}_{\mathbf{i}}$ is specified. Let's focus on two benchmark cases of the joint distribution - one with maximal positive linear correlation given marginals and one with maximal negative linear correlation given marginals. In the first case, lottery $\mathbf{y}$ takes the following form:

$$
\mathbf{y}=(-n x, 1 / 2 ; n x, 1 / 2)
$$

and in the second case lottery $\mathbf{y}$ takes the following form:

$$
\begin{array}{ll}
\mathbf{y}=(-x, 1 / 2 ; x, 1 / 2) & \text { if } n \text { odd } \\
\mathbf{y}=(0) & \text { if } n \text { even }
\end{array}
$$

Applying previous results we obtain:

perfect positive correlation given marginals:

$$
\begin{aligned}
& B\left(W, \sum_{i=1}^{n} \mathbf{x}_{\mathbf{i}}\right)<\sum_{i=1}^{n} B\left(W, \mathbf{x}_{\mathbf{i}}\right)=n B\left(W, \mathbf{x}_{\mathbf{i}}\right) \text { for } i \in\{1,2, \ldots, n\} \\
& S\left(W, \sum_{i=1}^{n} \mathbf{x}_{\mathbf{i}}\right)<\sum_{i=1}^{n} S\left(W, \mathbf{x}_{\mathbf{i}}\right)=n S\left(W, \mathbf{x}_{\mathbf{i}}\right) \text { for } i \in\{1,2, \ldots, n\}
\end{aligned}
$$

perfect negative correlation given marginals:

$$
\begin{aligned}
& n \text { odd } \begin{cases}B\left(W, \sum_{i=1}^{n} \mathbf{x}_{\mathbf{i}}\right)=B\left(W, \mathbf{x}_{\mathbf{i}}\right) & \text { for } i \in\{1,2, \ldots, n\} \\
S\left(W, \sum_{i=1}^{n} \mathbf{x}_{\mathbf{i}}\right)=S\left(W, \mathbf{x}_{\mathbf{i}}\right) & \text { for } i \in\{1,2, \ldots, n\}\end{cases} \\
& n \text { even }\left\{\begin{array}{l}
B\left(W, \sum_{i=1}^{n} \mathbf{x}_{\mathbf{i}}\right)=0 \\
S\left(W, \sum_{i=1}^{n} \mathbf{x}_{\mathbf{i}}\right)=0
\end{array}\right.
\end{aligned}
$$

\section{Concluding remarks}

In this paper for three different widely used risk attitudes classes, the altenative equivalent characterizations were presented, each corresponding to the properties of the following functions:

simple strategy

buying/selling price for a lottery

M. Lewandowski

CEJEME 5: 1-34 (2013) 
Risk Attitudes, Buying and Selling...

Pratt (1964), Arrow (1965) measures of risk aversion

Bernoulli utility function

These results may be useful both as technical help as well as a useful guide in empirical work. Not all of these results are new. It is however useful to put all of these results together and to offer a systematic proof of them. A simple strategy concept is a novel way to formalize existing intuition. Although parts of these results are well known in the literature, other parts turn out to be not sufficiently acknowledged and one can find statements in the literature which confirm it.

Another result in this paper is an extension to Pratt (1964) famous theorem on comparative risk aversion. It incorporates buying price as an alternative way to compare risk aversion across individuals. This result also might be useful both in theoretical and empirical work.

In section Additional results an interesting fact about CRRA utility function class is proved, namely that for any CRRA utility function except for the logarithm, which can be ignored as a limiting case, there exists an equivalent utility function that is homogeneous of some degree different than zero. Other results in this section develop further analysis of buying and selling price properties such as concavity and additivity.

\section{Acknowledgement}

I would like to thank Pascal Courty, Fernando Vega-Redondo, Roberto Serrano, Robert Sugden, and audiences of many seminars for help, support, advice and guidance.

\section{References}

Arrow, K. J. (1965). Aspects of the Theory of Risk-bearing. Yrjo Jahnsson Lectures, Helsinki.

Aumann R. J., Kurz M. (1977). Power and taxes. Econometrica 45 (5), 1137-1161.

Aumann R. J., Serrano R. (2008). An economic index of riskiness. Journal of Political Economy 116, 810-836

Barberis N., Huang M. (2009). Preferences with frames: a new utility specification that allows for the framing of risks, Journal of Economic Dynamics and Control

Foster, D. and S. Hart (2007), An operational measure of riskiness.

LeRoy, S. F. and J. Werner (2001). Principles of Financial Economics. Cam-bridge University Press. 
Lewandowski, M. (2011). Buying and selling price for risky lotteries and ex- pected utility theory with gambling wealth. http://ssrn.com/abstract $=2189400$.

Pratt, J. W. (1964). Risk aversion in the small and in the large. Econometrica 32, $122-136$.

Raiffa, H. (1968). Decision Analysis: Introductory Lectures on Choices Under Uncertainty. Addison-Wesley 22 
Risk Attitudes, Buying and Selling...

\section{Proofs}

A couple of lemmas will be necessary.

Lemma 3. For any lottery $\mathbf{x}$ and any wealth level $W$, the following holds:

$$
\begin{aligned}
S[W, \mathbf{x}-B(W, \mathbf{x})] & =0 \\
S[W-B(W, \mathbf{x}), \mathbf{x}] & =B(W, \mathbf{x}) \\
B[W+S(W, \mathbf{x}), \mathbf{x}] & =S(W, \mathbf{x})
\end{aligned}
$$

Proof. First equation (20) will be proved. Define $\mathbf{y}=\mathbf{x}-B(W, \mathbf{x})$. Using equations (2) and (1)

$$
\begin{aligned}
U(W) & =\mathrm{E} U[W+(\mathbf{x}-B(W, \mathbf{x}))] \\
& =\mathrm{E} U[W+\mathbf{y}] \\
& =U[W+S(W, \mathbf{y})] \\
& =U[W+S(W, \mathbf{x}-B(W, \mathbf{x}))]
\end{aligned}
$$

And condition (20) follows. Now equation (21) will be proved. Define $V=W-B(W, \mathbf{x})$. Using equations (2) and (1)

$$
\begin{aligned}
U(W) & =\mathrm{E} U[(W-B(W, \mathbf{x}))+\mathbf{x}] \\
& =\mathrm{E} U[V+\mathbf{x}] \\
& =U[V+S(V, \mathbf{x})] \\
& =U[W-B(W, \mathbf{x})+S(W-B(W, \mathbf{x}), \mathbf{x})]
\end{aligned}
$$

And condition (21) follows. Now equation (22) will be proved. Define $V=W+S(W, \mathbf{x})$. Using equations (2) and (1)

$$
\begin{aligned}
\mathrm{E} U(W+\mathbf{x}) & =U[W+S(W, \mathbf{x})] \\
& =U(V) \\
& =\mathrm{E} U[V+\mathbf{x}-B(V, \mathbf{x})] \\
& =\mathrm{E} U[W+S(W, \mathbf{x})+\mathbf{x}-B(W+S(W, \mathbf{x}), \mathbf{x})]
\end{aligned}
$$

And so condition (22) is proved.

Lemma 4. For any non-degenerate lottery $\mathbf{x}$ and any wealth $W$ such that buying and selling price exist, $S(W, \mathbf{x})$ and $B(W, \mathbf{x})$ lie in the interval $(\min (\mathbf{x}), \mathrm{E}(\mathbf{x}))$. For a degenerate lottery $\mathbf{x}, S(W, \mathbf{x})=B(W, \mathbf{x})=x$.

Proof. Notice first that for degenerate lottery $\mathbf{x}=x$, equations (1) and (2) imply the following:

$$
\begin{aligned}
W+S(W, x) & =W+x \\
W+x-B(W, x) & =W
\end{aligned}
$$


And so $S(W, x)=B(W, x)=x$. From now on we shall focus on a non-degenerate lottery $\mathbf{x}$. The lemma will be proved for selling price only. For buying price the proof is similar. For simplicity let's define $S \equiv S(W, \mathbf{x})$. The proof is by contradiction. Suppose $\min _{i \in\{1, \ldots, n\}} x_{i} \geq S$. Then:

$$
U\left(W+x_{i}\right) \geq U\left(W+\min _{i \in\{1, \ldots, n\}} x_{i}\right) \geq U(W+S)
$$

with strict inequality for any $x_{i} \neq \min _{i \in\{1, \ldots, n\}} x_{i}$. Since lottery $\mathbf{x}$ is non-degenerate there exists at least one $x_{i} \neq \min _{i \in\{1, \ldots, n\}} x_{i}$ Hence

$$
\sum_{i=1}^{n} p_{i} U\left(W+x_{i}\right)>U(W+S)
$$

So $S$ cannot be the selling price - a contradiction.

Suppose now that $S \geq \mathrm{E}[\mathbf{x}]$. By strict Jensen's inequality:

$$
\mathrm{E} U[W+\mathbf{x}]<U[W+\mathrm{E}[\mathbf{x}]] \leq U(W+S)
$$

So $S$ cannot be the selling price - a contradiction.

Lemma 5. Given a twice continuously differentiable utility function $U$, the following holds:

$$
A R A(W)=\lim _{h \rightarrow 0^{+}} \frac{4}{h}\left(p(W, h)-\frac{1}{2}\right)
$$

where $A R A(W)=-\frac{U^{\prime \prime}(W)}{U^{\prime}(W)}$ is an absolute risk aversion coefficient and $p(W, h)$ is a probability premium defined implicitly by:

$$
p(W, h) U(W+h)+(1-p(W, h)) U(W-h)=U(W)
$$

Moreover, for $h=\lambda W$, the following is obtained:

$$
R R A(W)=\lim _{\lambda \rightarrow 0^{+}} \frac{4}{\lambda}\left(p(W, \lambda W)-\frac{1}{2}\right)
$$

Proof. Rewriting equation (24) by using second order Taylor expansion of $U$ around $W$, the following is obtained for small $h$ :

$$
\begin{aligned}
& p(W, h)\left[U(W)+U^{\prime}(W) h+\frac{1}{2} U^{\prime \prime}(W) h^{2}\right] \\
& +(1-p(W, h))\left[U(W)-U^{\prime}(W) h+\frac{1}{2} U^{\prime \prime}(W) h^{2}\right] \approx U(W)
\end{aligned}
$$

And after simplifying:

$$
\frac{1}{2} U^{\prime \prime}(W) h+U^{\prime}(W)(2 p(W, h)-1) \approx 0
$$

M. Lewandowski

CEJEME 5: 1-34 (2013) 
Risk Attitudes, Buying and Selling...

Or

$$
A R A(W)=\lim _{h \rightarrow 0^{+}} \frac{4}{h}\left(p(W, h)-\frac{1}{2}\right)
$$

as was to be shown. Set $h=\lambda W$ and equation (25) immediately follows.

\subsection{Proof of proposition 4.1}

The proof will be split into two lemmas.

Lemma 6. Simple strategy of an individual is wealth invariant if and only if he exhibits CARA.

Proof. Similar technique to that used in this proof was used in Aumann and Kurz (1977).

If the decision maker's Bernoulli utility function is $U$, then wealth-invariant strategy can be described alternatively by the following condition:

$$
\mathrm{E} U\left(W_{1}+\mathbf{x}\right) \geq U\left(W_{1}\right) \Longleftrightarrow \mathrm{E} U\left(W_{2}+\mathbf{x}\right) \geq U\left(W_{2}\right), \quad \forall W_{1}, W_{2}
$$

(Necessity)

CARA utility functions take the following form $U(x)=A e^{-a x}+B$, where $A<0$, $a \geq 0$ and $B$ are arbitrary constants (such that utility is strictly increasing). It is straightforward to verify that CARA utility functions correspond to wealth-invariant strategies.

(Sufficiency)

Given utility function $U$, consider two lotteries $\mathbf{x}_{\mathbf{i}} \equiv\left(h, p\left(W_{i}, h\right) ;-h, 1-p\left(W_{i}, h\right)\right)$, where $W_{i}, h>0, i \in\{1,2\}$, such that:

$$
\mathrm{E} U\left(W_{i}+\mathbf{x}_{\mathbf{i}}\right)=U\left(W_{i}\right)
$$

Contrary to what is to be shown, assume that $A\left(W_{1}\right)>A\left(W_{2}\right)$, where $A(W)$ is absolute risk aversion function. By lemma 5 equation (23), for $h$ sufficiently small we know that $p\left(W_{1}, h\right)>p\left(W_{2}, h\right)$. Let $q$ be between the two probability premiums: $p\left(W_{1}, h\right)>q>p\left(W_{2}, h\right)$. Let's define another lottery $\mathbf{y} \equiv(h, q ;-h, 1-q)$. By definition of a probability premium utility function $U$ "rejects" lottery y at wealth $W_{1}$ and "accepts" it at wealth $W_{2}$, which contradicts wealth invariance.

Lemma 7. Given any $W_{1}$ and $W_{2}$, the following holds:

$$
B\left(W_{1}, \mathbf{x}\right)=S\left(W_{2}, \mathbf{x}\right) \forall W_{1}, W_{2} \quad \Longleftrightarrow \quad \text { the strategy is wealth - invariant }
$$

where $B$ and $S$ are buying and selling price function, respectively ${ }^{15}$.

\footnotetext{
${ }^{15}$ Notice that the condition on the left-hand side of the above equivalence is the same as condition iii. in the statement of proposition 4.1.
} 
Proof. First step) First, it will be proved that $S$ is independent of $W$ iff the strategy is wealth-invariant.

(Neccessity)

If the strategy is wealth invariant then by condition (26) it follows that:

$$
\mathrm{E} U\left(W_{1}+\mathbf{x}\right)=U\left(W_{1}\right) \Longleftrightarrow \mathrm{E} U\left(W_{2}+\mathbf{x}\right)=U\left(W_{2}\right), \quad \forall W_{1}, W_{2}
$$

Let's denote $S\left(W_{i}, \mathbf{x}\right)=S_{i} i \in\{1,2\}$ and assume $W_{1} \neq W_{2}$. From the definition of selling price:

$$
\mathrm{E} U\left(W_{1}+\mathbf{x}\right)=U\left(W_{1}+S_{1}\right)
$$

Let's define $V_{1}=W_{1}+S_{1}, V_{2}=W_{2}+S_{2}$ and $\mathbf{y}=\mathbf{x}-S_{1}$. By equation (28) we know that:

$$
\mathrm{E} U\left(V_{1}+\mathbf{y}\right)=U\left(V_{1}\right) \Longleftrightarrow \mathrm{E} U\left(V_{2}+\mathbf{y}\right)=U\left(V_{2}\right)
$$

Hence by substituting $\mathbf{y}=\mathbf{x}-S_{1}$ and $V_{2}=W_{2}+S_{2}$ into the RHS of the above condition:

$$
\mathrm{E} U\left(W_{2}+S_{2}+\mathbf{x}-S_{1}\right)=U\left(W_{2}+S_{2}\right)
$$

And by definition of $S_{2}$ we know that $S_{1}$ has to be equal to $S_{2}: S\left(W_{1}, \mathbf{x}\right)=S\left(W_{2}, \mathbf{x}\right)$ (Sufficiency)

Suppose that the strategy is not wealth-invariant. Then:

$$
\exists W_{1}, W_{2}, \mathbf{x}: \quad \mathrm{E} U\left(W_{1}+\mathbf{x}\right) \geq U\left(W_{1}\right) \text { and } \mathrm{E} U\left(W_{2}+\mathbf{x}\right)<U\left(W_{2}\right),
$$

Notice that by strict monotonicity of $U$ it follows that $S\left(W_{1}, \mathbf{x}\right) \geq 0$ and $S\left(W_{2}, \mathbf{x}\right)<0$ which contradicts the fact that $S$ is wealth-invariant.

Second step) Now it is sufficient to prove that $S$ is equal to $B$ iff $S$ is independent of $W$.

(Necessity)

If $S$ is independent of $W$ then $S(W, \mathbf{x})=S\left(W^{\prime}, \mathbf{x}\right)$ for any $W$ and $W^{\prime}$. Take $W^{\prime}=W-B(W, \mathbf{x})$ and any $W$. Then from lemma 3 equation (21) we have that: $B(W, \mathbf{x})=S(W-B(W, \mathbf{x}), \mathbf{x})$. And from the fact that $S$ is independent of wealth, we obtain $S(W, \mathbf{x})=B(W, \mathbf{x})$. Since $W$ was arbitrary $B$ is also wealth independent and necessity is proved.

(Sufficiency)

Take any $W$ and fix it. Suppose $S\left(W^{\prime}, \mathbf{x}\right)=B(W, \mathbf{x})$ for any $W^{\prime}$. Then obviously $S$ has to be independent of wealth. This finishes the proof.

Taken together lemma 6 and lemma 7 establish proposition 4.1.

\subsection{Proof of proposition $\mathbf{4 . 2}$}

The proof is split into three parts. The first part is the following.

Lemma 8. If simple strategy of an individual is of "wealthier-accept more" type then he exhibits DARA.

M. Lewandowski

CEJEME 5: 1-34 (2013) 
Risk Attitudes, Buying and Selling...

Proof. The contrapositive of the above statement will be proved. Suppose $A\left(W_{1}\right) \leq A\left(W_{2}\right)$ for any $W_{1}<W_{2}$. If $A\left(W_{1}\right)=A\left(W_{2}\right)$ then by proposition 4.1 simple strategy is wealth-invariant so it cannot be of "wealthier-accept more" type. Suppose then that $A\left(W_{1}\right)<A\left(W_{2}\right)$ for some given $W_{1}<W_{2}$. Consider two lotteries: $\mathbf{x}_{i}=\left(h, p\left(W_{i}, h\right) ;-h, 1-p\left(W_{i}, h\right)\right), i=\{1,2\}$ such that:

$$
\mathrm{E} U\left(W_{i}+\mathbf{x}_{\mathbf{i}}\right)=U\left(W_{i}\right)
$$

Then by lemma $5, p\left(W_{1}, h\right)<p\left(W_{2}\right)$ for $h$ small enough. We construct another lottery $\mathbf{y}=(h, q ;-h, 1-q)$ such that $p\left(W_{1}, h\right)<q<p\left(W_{2}\right)$. Then by construction $U$ "rejects" lottery y at wealth level $W_{2}$ and "accepts" it at wealth level $W_{1}$ which means that the strategy cannot be of "wealthier-accept more" type.

The second part of the proof consists of three results. The following result is corollary to Pratt (1964) theorem. The proof is due to LeRoy and Werner (2001).

Corollary 1. For a strictly increasing and twice differentiable utility function $U$ with continuous second derivative, the following holds:

$S(W, \mathbf{x})$ is increasing/constant/decreasing in $W$ for every $\mathbf{x}$ iff $A(W)$ is decreasing/constant/increasing in $W$

where $A(W)$ denotes absolute risk aversion as a function of $W$.

Proof. We will prove only the increasing $S$ case. The rest is similar. Given a utility function $U_{1}(W)$, define another utility function $U_{2}(W)=U_{1}(W+\Delta)$, where $\Delta \geq 0$. We can then apply Pratt (1964) theorem: $S_{1}(W, \mathbf{x})<S_{2}(W, \mathbf{x})=S_{1}(W+\Delta, \mathbf{x}) \Leftrightarrow$ $\Leftrightarrow A_{1}(W)>A_{2}(W)=A_{1}(W+\Delta)$. Since $\Delta$ was arbitrary the corollary is proved.

Lemma 9. If utility function is of DARA type, then the following holds:

$$
B(W, \mathbf{x})>0 \Longleftrightarrow B(W, \mathbf{x})<S(W, \mathbf{x})
$$

Proof. Using equation (21) of lemma 3 and corollary 1 to Pratt (1964) theorem, the following is obtained:

$$
0<B(W, \mathbf{x})=S[W-B(W, \mathbf{x}), \mathbf{x}]<S(W, \mathbf{x})
$$

Lemma 10. For a strictly increasing and twice differentiable utility function $U(\cdot)$ with continuous second derivative, the following holds:

- $B(W, \mathbf{x})$ is increasing/constant/decreasing in $W$ for every $\mathbf{x}$ iff $A(W)$ is decreasing/constant/increasing in $W$

where $A(W)$ denotes absolute risk aversion as a function of $W$.

Proof. By corollary 1, it suffices to show the following: 
$S(W, \mathbf{x})$ is increasing/constant/decreasing in $W$ for any $\mathbf{x}$ iff $B(W, \mathbf{x})$ is increasing/constant/decreasing in $W$ for any $\mathbf{x}$.

Only the "increasing part" we will shown. The rest is similar.

$(\Rightarrow)$ The proof is by contradiction. Take $\mathbf{x}$ such that, if $W_{1}<W_{2}$ then $B\left(W_{1}, \mathbf{x}\right) \geq B\left(W_{2}, \mathbf{x}\right)$. Fix this $\mathbf{x}$. Since $S(W, \mathbf{x})$ is increasing in $W$ for any $\mathbf{x}$, we have:

$$
S\left[W_{2}, \mathbf{x}-B\left(W_{1}, \mathbf{x}\right)\right]>S\left[W_{1}, \mathbf{x}-B\left(W_{1}, \mathbf{x}\right)\right]=S\left[W_{2}, \mathbf{x}-B\left(W_{2}, \mathbf{x}\right)\right]=0
$$

where we made use of lemma 3 equation (20). By lemma 15, equation (38), we obtain from above::

$$
S\left(W_{2}-B\left(W_{1}, \mathbf{x}\right), \mathbf{x}\right)-B\left(W_{1}, \mathbf{x}\right)>S\left(W_{2}-B\left(W_{2}, \mathbf{x}\right), \mathbf{x}\right)-B\left(W_{2}, \mathbf{x}\right)
$$

And hence after rearranging and using the assumption:

$$
\begin{aligned}
0 & \leq B\left(W_{1}, \mathbf{x}\right)-B\left(W_{2}, \mathbf{x}\right) \\
& <S\left(W_{2}-B\left(W_{1}, \mathbf{x}\right), \mathbf{x}\right)-S\left(W_{2}-B\left(W_{2}, \mathbf{x}\right), \mathbf{x}\right) \\
& \leq 0
\end{aligned}
$$

Which is a contradiction and hence the "if" part of the lemma is proved.

$(\Leftarrow)$ Again by contradiction. Take $\mathbf{x}$ such that, if $W_{1}<W_{2}$ then $S\left(W_{1}, \mathbf{x}\right) \geq S\left(W_{2}, \mathbf{x}\right)$. Fix this $\mathbf{x}$. By lemma 3 equation (21) we have:

$$
\begin{aligned}
B\left(W_{1}, \mathbf{x}\right) & =S\left(W_{1}-B\left(W_{1}, \mathbf{x}\right), \mathbf{x}\right) \\
& \geq S\left(W_{2}-B\left(W_{1}, \mathbf{x}\right), \mathbf{x}\right) \\
& \geq S\left(W_{2}-B\left(W_{2}, \mathbf{x}\right), \mathbf{x}\right)=B\left(W_{2}, \mathbf{x}\right)
\end{aligned}
$$

where the first inequality follows from our assumption and second inequality follows from the fact that $B(W, \mathbf{x})$ is increasing in $W$ for any $\mathbf{x}$. Hence $B\left(W_{1}, \mathbf{x}\right) \geq B\left(W_{2}, \mathbf{x}\right)$ - a contradiction.

Combining corollary 1 and lemmas 9 and 10, it is established that when utility is DARA then buying and selling price are increasing in $W$ and that

$$
B(W, \mathbf{x})>0 \Longleftrightarrow B(W, \mathbf{x})<S(W, \mathbf{x})
$$

The third step in proving proposition 4.2 is the following:

Lemma 11. If selling price is increasing in $W$ then strategy is of "wealthier-accept more" type.

Proof. Let's focus on lotteries the acceptance of which, given preferences, depends on wealth level. That is there exists two different wealth levels $W_{1}, W_{2}$ such that $S\left(W_{1}, \mathbf{x}\right)<0$ (reject) and $S\left(W_{2}, \mathbf{x}\right)>0$ (accept). Since $S$ is increasing in $W$, it must be that $W_{1}<W_{2}$.

M. Lewandowski

CEJEME 5: 1-34 (2013) 
Risk Attitudes, Buying and Selling...

\subsection{Proof of proposition $\mathbf{4 . 3}$}

Now proposition 4.3 will be proved. The proof is split into three lemmas.

Lemma 12. Simple strategy of an individual is scale invariant if and only if he exhibits CRRA.

$C R R A$. If the decision maker's Bernoulli utility function is $U$, then homogeneous strategy can be described alternatively by the following condition:

$$
\mathrm{E} U(W+\mathbf{x}) \geq U(W) \Longleftrightarrow \mathrm{E} U(\lambda W+\lambda \mathbf{x}) \geq U(\lambda W), \quad \forall \lambda>0
$$

(Necessity)

All CRRA utility functions belong to the following family: $U(x)=A x^{a}+B$, where $A>0,1 \neq a \geq 0$ and $B$ are arbitrary constants (such that utility is strictly increasing) and $U(x)=A \log x+B$ for $a=1$, where $A \geq 0$ and $B$ are arbitrary constants. It is easy to verify that indeed, CRRA class of utility functions represents homogeneous strategies.

(Sufficiency)

Given utility function $U$, consider lottery $\mathbf{x} \equiv(W h, p(W, h) ;-W h, 1-p(W, h))$, where $W, h>0$, such that:

$$
\mathrm{E} U(W+\mathbf{x})=U(W)
$$

Contrary to what is to be shown, assume that $R(\lambda W)>R(W)$, for $\lambda>0$ and $\lambda \neq 1$. Define $\mathbf{x}^{\prime} \equiv(W h, p(\lambda W, h) ;-W h, 1-p(\lambda W, h))$, such that:

$$
\mathrm{E} U\left(\lambda W+\lambda \mathbf{x}^{\prime}\right)=U(\lambda W)
$$

For $h$ sufficiently small, by proposition 5 equation (25), we know that: $p(\lambda W, h)>p(W, h)$. Therefore, one can find $q$ such that: $p(\lambda W, h)>q>p(W, h)$. Define $\mathbf{y} \equiv(W h, q ;-W h,(1-q))$.

By equation (32), since $p(\lambda W, h)>q$ :

$$
\mathrm{E} U(\lambda W+\lambda \mathbf{x})<U(\lambda W)
$$

By equation (31), and since $q>p(W, h)$, we have:

$$
\mathrm{E} U(W+\mathbf{y})>U(W)
$$

which is a contradiction.

Lemma 13. Given any $\lambda>0$, the following holds:

$$
\begin{aligned}
& S(\lambda W, \lambda \mathbf{x})=\lambda S(W, \mathbf{x}) \\
& B(\lambda W, \lambda \mathbf{x})=\lambda B(W, \mathbf{x})
\end{aligned} \quad \Longleftrightarrow \quad \text { strategy is homogeneous. }
$$

where $B$ and $S$ are buying and selling price function, respectively. 
Proof. (Necessity)

It will be proved only for selling price $S$. For buying price proof is similar. To avoid heavy notation, let's define $S_{\lambda} \equiv S(\lambda W, \lambda \mathbf{x})$ and $S \equiv S(W, \mathbf{x})$. If the decision maker's Bernoulli utility function is $U$, then scale-invariant strategy can be described alternatively by the following condition:

$$
\mathrm{E} U(W+\mathbf{x})=U(W) \quad \Longleftrightarrow \quad \mathbf{E} U(\lambda W+\lambda \mathbf{x})=U(\lambda W), \quad \forall \lambda>0
$$

By definition of $S_{\lambda}$ :

$$
\mathrm{E} U(\lambda W+\lambda \mathbf{x})=U\left(\lambda W+S_{\lambda}\right)
$$

Define $V \equiv W+\frac{1}{\lambda} S_{\lambda}$ and $\mathbf{y} \equiv \mathbf{x}-\frac{1}{\lambda} S_{\lambda}$. Then we can rewrite the above equation as:

$$
\mathrm{E} U(\lambda V+\lambda \mathbf{y})=U(\lambda V)
$$

By condition (33), we have:

$$
\mathbf{E} U(V+\mathbf{y})=U(V) \Longleftrightarrow \mathbf{E} U(\lambda V+\lambda \mathbf{y})=U(\lambda V), \quad \forall \lambda>0
$$

And hence

$$
\mathrm{E} U(W+\mathbf{x})=U\left(W+\frac{1}{\lambda} S_{\lambda}\right)
$$

So, by definition of $S$, it has to be that: $\frac{1}{\lambda} S_{\lambda}=S$ or by returning to the original notation:

$$
S(\lambda W, \lambda \mathbf{x})=\lambda S(W, \mathbf{x}), \quad \forall \lambda>0
$$

(Sufficiency)

Suppose the strategy is not homogeneous. Then there exists $\lambda>0$, such that:

$$
\mathrm{E} U(W+\mathbf{x}) \geq U(W) \text { and } \mathrm{E} U(\lambda W+\lambda \mathbf{x})<U(\lambda W)
$$

It follows that $S(W, \mathbf{x}) \geq 0$ and $S(\lambda W, \lambda \mathbf{x})<0$, by strict monotonicity of $U$ and the fact that $\lambda$ is positive. Hence it is not possible that this $\lambda, S(\lambda W, \lambda \mathbf{x})=\lambda S(W, \mathbf{x})$. A contradiction.

Lemma 14. Simple strategy is scale-invariant if and only if buying and selling return prices for any multiplicative lottery are independent from wealth and equal i.e.

$$
b(W, \mathbf{h})=s(W, \mathbf{h})=C_{\beta}, \forall W
$$

where $\beta$ is relative risk aversion coefficient and $C_{\beta}$ takes real values and depends only on $\beta$. Additionally,

$$
\begin{aligned}
& b(W, \lambda \mathbf{h})=\lambda b(W, \mathbf{h}) \\
& s(W, \lambda \mathbf{h})=\lambda s(W, \mathbf{h})
\end{aligned}
$$


Risk Attitudes, Buying and Selling...

Proof. If a strategy is scale-invariant, it is easy to see from the definitions of buying and selling return prices (11) and (10) that all the conditions of $b(W, \mathbf{h})$ and $s(W, \mathbf{h})$ above are satisfied. Similarly in the other direction, it is easy to see that if the conditions above are satisfied, the strategy must be scale-invariant due to the nature of multiplicative gambles and the way they are handled in conditions (11) and (10) defining buying and selling return prices.

\subsection{Proof of proposition 4.4}

We will first prove two additional lemmas and then the proposition. The first proposition states that buying price exhibits the so called delta property whereas selling price in general does not.

Lemma 15. For any lottery $\mathbf{x}$ and any wealth level $W$ and for $\Delta \in \mathbb{R}$, the following holds:

$$
\begin{aligned}
S(W, \mathbf{x}+\Delta) & =S(W+\Delta, \mathbf{x})+\Delta \\
B(W, \mathbf{x}+\Delta) & =B(W, \mathbf{x})+\Delta
\end{aligned}
$$

Proof. From the definition of selling price:

$$
\begin{aligned}
\mathrm{E} U(W+\mathbf{x}+\Delta) & =U[W+S(W, \mathbf{x}+\Delta)] \\
& =U[W+\Delta+S(W+\Delta, \mathbf{x})]
\end{aligned}
$$

And hence equation (38) holds. From the definition of buying price:

$$
\begin{aligned}
U(W) & =\mathrm{E} U[W+(\mathbf{x}+\Delta)-B(W, \mathbf{x}+\Delta)] \\
& =\mathrm{E} U[W+\mathbf{x}-B(W, \mathbf{x})]
\end{aligned}
$$

And hence equation (39) holds.

A function $F(W, \mathbf{x})$ exhibits delta property if $F(W, \mathbf{x}+\Delta)=F(W, \mathbf{x})+\Delta$ for $\Delta \in \mathbb{R}$. Thus, the buying price exhibits delta property, while selling price in general does not - see equations (38) and (39) above. There is however a special class of utility functions for which selling price exhibits the delta property, namely the class of constant absolute risk aversion (CARA). Notice from equation (38) that selling price would obey the delta property if only $S(W+\Delta, \mathbf{x})=S(W, \mathbf{x})$ for $\Delta \in \mathbb{R}$. That means that selling price would be independent of wealth level $W$. And indeed, as could easily be checked, selling price for CARA utility is independent of wealth. In fact, the stronger result by Raiffa (1968) holds- CARA utility is equivalent to selling price exhibiting the delta property.

Lemma 16. For differentiable DARA utility functions, given any non-degenerate lottery $\mathbf{x}$ and any wealth level $W$, the following holds:

$$
0<\frac{\partial B(W, \mathbf{x})}{\partial W}<1
$$


Proof. From the definition of buying, selling price and the fact that they are both increasing in wealth, it follows that:

$$
\frac{\partial B(W, \mathbf{x})}{\partial W}=\frac{\mathrm{E} U^{\prime}(W+\mathbf{x}-B(W, \mathbf{x}))-U^{\prime}(W)}{\mathrm{E} U^{\prime}(W+\mathbf{x}-B(W, \mathbf{x}))}>0
$$

The result follows.

Proposition 7.1. For two different utility functions $U_{1}(\cdot)$ and $U_{2}(\cdot)$ with decreasing absolute risk aversion (DARA), any wealth level $W$ and any non-degenerate random variable $\mathbf{x}$ with bounded values, let's define corresponding selling and buying prices $S_{1}(W, \mathbf{x}), B_{1}(W, \mathbf{x})$ and $S_{2}(W, \mathbf{x}), B_{2}(W, \mathbf{x})$. The following equivalence holds:

$$
\begin{gathered}
\forall W \forall \mathbf{x}: \exists \delta>0\left|x_{i}\right|<\delta \forall i \in\{1, \ldots, n\} \\
S_{1}(W, \mathbf{x})>S_{2}(W, \mathbf{x}) \Longleftrightarrow B_{1}(W, \mathbf{x})>B_{2}(W, \mathbf{x})
\end{gathered}
$$

Proof. $(\Rightarrow)$ By contradiction. Fix $\mathbf{x}$ with bounded values and $W$ for which the following holds: $B_{1}(W, \mathbf{x}) \leq B_{2}(W, \mathbf{x})$. By lemma 3 equation (21), we obtain:

$$
\begin{aligned}
S_{1}\left(W-B_{1}(W, \mathbf{x}), \mathbf{x}\right) & \leq S_{2}\left(W-B_{2}(W, \mathbf{x}), \mathbf{x}\right) \\
& \leq S_{2}\left(W-B_{1}(W, \mathbf{x}), \mathbf{x}\right)
\end{aligned}
$$

where the second inequality follows from the fact that $S_{2}$ is increasing in the first argument and $B_{1}(W, \mathbf{x}) \leq B_{2}(W, \mathbf{x})$. This is a contradiction since we have found $V=W-B_{1}(W, \mathbf{x})$ and $\mathbf{x}$ for which $S_{1}(V, \mathbf{x}) \leq S_{2}(V, \mathbf{x})$. Thus the first part of the proposition is proved.

$(\Leftarrow)$ By contradiction. Suppose $S_{1}(V, \mathbf{x}) \leq S_{2}(V, \mathbf{x})$ for some $V$ and some $\mathbf{x}$ with bounded values. Take lottery $\mathbf{y}: \mathbf{y}=\mathbf{x}$. Take wealth level $W: V=W-B_{1}(W, \mathbf{x})$. Such wealth level exists for any $V \in \mathbb{R}$. To prove this, let's define a function $W$ : $\mathbb{R} \rightarrow \mathbb{R}$ taking values $V(W)=W-B_{1}(W, \mathbf{x})$. This function is a bijection and takes values in the whole real line $(-\infty,+\infty)$. First, by the fact that lottery $\mathbf{x}$ has bounded values we know by lemma 5 that $B_{1}(W, \mathbf{x})$ is also bounded. On the other hand $W$ is not bounded. Hence, $V(W)$ is also not bounded. Second, by lemma 16, we know that $\frac{\partial B_{1}(W, \mathbf{x})}{\partial W}<1$ and thus $V^{\prime}(W)>0$. Therefore, $V(W)$ is both surjection and injection and hence bijection. This proves that for any $V \in \mathbb{R}$, there exists a unique $W$ such that $V=W-B_{1}(W, \mathbf{x})$. If this holds for any $V$, then it holds for some $V$ such that given some $\mathbf{x}$, the following holds $S_{1}(V, \mathbf{x}) \leq S_{2}(V, \mathbf{x})$. We will now show that for lottery $\mathbf{y}$ and wealth level $W, B_{1}(W, \mathbf{y}) \leq B_{2}(W, \mathbf{y})$. In fact, using lemma 3 equation

CEJEME 5: 1-34 (2013) 
(21):

$$
\begin{aligned}
B_{1}(W, \mathbf{y}) & =B_{1}(W, \mathbf{x}) \\
& =S_{1}\left(W-B_{1}(W, \mathbf{x}), \mathbf{x}\right) \\
& =S_{1}(V, \mathbf{x}) \\
& \leq S_{2}(V, \mathbf{x}) \\
& =S_{2}\left(W-B_{1}(W, \mathbf{x}), \mathbf{x}\right) \\
& <S_{2}\left(W-B_{2}(W, \mathbf{x}), \mathbf{x}\right) \\
& =B_{2}(W, \mathbf{x})=B_{2}(W, \mathbf{y})
\end{aligned}
$$

where the last inequality holds due to the fact that $B_{1}(W, \mathbf{x})>B_{2}(W, \mathbf{x})$ for all $W$ and for all $\mathbf{x}$ with bounded values and $S_{2}$ is increasing in the first argument. A contradiction. Hence the proposition is proved.

\subsection{Proof of proposition $\mathbf{5 . 5}$}

The proof is split into two lemmas.

Lemma 17. For any $W_{1} \neq W_{2}$ and for all $\theta \in(0,1)$ and any non-degenerate lottery $\mathbf{x}$, the following holds for constant relative risk aversion utility function:

$$
\begin{array}{r}
S\left(\theta W_{1}+(1-\theta) W_{2}, \mathbf{x}\right)>\theta S\left(W_{1}, \mathbf{x}\right)+(1-\theta) S\left(W_{2}, \mathbf{x}\right) \\
B\left(\theta W_{1}+(1-\theta) W_{2}, \mathbf{x}\right)>\theta B\left(W_{1}, \mathbf{x}\right)+(1-\theta) B\left(W_{2}, \mathbf{x}\right)
\end{array}
$$

provided that both sides are well defined.

Proof. We will show that for all $\theta \in(0,1)$ and for all $W_{1} \neq W_{2}$, the following holds:

$$
S\left(\theta W_{1}+(1-\theta) W_{2}, \mathbf{x}\right)>\theta S\left(W_{1}, \mathbf{x}\right)+(1-\theta) S\left(W_{2}, \mathbf{x}\right)
$$

Let's define $S_{i}=S\left(W_{i}, \mathbf{x}\right)$, where $i=1,2$. By the property of homogeneity the following follows from the definition:

$$
\mathrm{E} U\left(\frac{\theta W_{1}+(1-\theta) W_{2}+\mathbf{x}}{\theta\left(W_{1}+S_{1}\right)+(1-\theta)\left(W_{2}+S_{2}\right)}\right)>1
$$

Define $\lambda=\frac{\theta\left(W_{1}+S_{1}\right)}{\theta\left(W_{1}+S_{1}\right)+(1-\theta)\left(W_{2}+S_{2}\right)}$. Then, by concavity of $U$ :

$$
\begin{aligned}
1 & =\lambda \mathrm{E} U\left(\frac{W_{1}+\mathbf{x}}{W_{1}+S_{1}}\right)+(1-\lambda) \mathrm{E} U\left(\frac{W_{2}+\mathbf{x}}{W_{2}+S_{2}}\right) \\
& <\mathrm{E} U\left(\lambda \frac{W_{1}+\mathbf{x}}{W_{1}+S_{1}}+(1-\lambda) \frac{W_{2}+\mathbf{x}}{W_{2}+S_{2}}\right) \\
& =\mathrm{E} U\left(\frac{\theta W_{1}+(1-\theta) W_{2}+\mathbf{x}}{\theta\left(W_{1}+S_{1}\right)+(1-\theta)\left(W_{2}+S_{2}\right)}\right)
\end{aligned}
$$


Similarly for the buying price case, define $B_{i}=B\left(W_{i}, \mathbf{x}\right)$, where $i=1,2$. We will prove that:

$$
\mathrm{E} U\left(\frac{\theta\left(W_{1}-B_{1}\right)+(1-\theta)\left(W_{2}-B_{2}\right)+\mathbf{x}}{\theta W_{1}+(1-\theta) W_{2}}\right)>1
$$

Define $\lambda=\frac{\theta W_{1}}{\theta W_{1}+(1-\theta) W_{2}}$

$$
\begin{aligned}
1 & =\lambda \mathrm{E} U\left(\frac{W_{1}-B_{1}+\mathbf{x}}{W_{1}}\right)+(1-\lambda) \mathrm{E} U\left(\frac{W_{2}-B_{2}+\mathbf{x}}{W_{2}}\right) \\
& <\mathrm{E} U\left(\lambda \frac{W_{1}-B_{1}+\mathbf{x}}{W_{1}}+(1-\lambda) \frac{W_{2}-B_{2}+\mathbf{x}}{W_{2}}\right) \\
& =\mathrm{E} U\left(\frac{\theta\left(W_{1}-B_{1}\right)+(1-\theta)\left(W_{2}-B_{2}\right)+\mathbf{x}}{\theta W_{1}+(1-\theta) W_{2}}\right)
\end{aligned}
$$

Lemma 18. For CRRA utility function, the following holds $\forall \theta \in(0,1)$

$$
\begin{array}{r}
S(\theta W, \mathbf{x})+S((1-\theta) W, \mathbf{y})<S(W, \mathbf{x}+\mathbf{y}) \\
B(\theta W, \mathbf{x})+B((1-\theta) W, \mathbf{y})<B(W, \mathbf{x}+\mathbf{y})
\end{array}
$$

provided that both sides are well defined.

Proof. Let's start with the selling price. Define $S_{1}=S(\theta W, \mathbf{x})$ and $S_{2}=S((1-\theta) W, \mathbf{y})$. The proof is similar to the proof of concavity of $S$ and $B$ in $W$. Define $\lambda=\frac{\theta W+S_{1}}{W+S_{1}+S_{2}}$. Note that $1-\lambda=\frac{(1-\theta) W}{W+S_{1}+S_{2}}$. Then it follows from homogeneity and the definition that:

$$
\begin{aligned}
1 & =\lambda \mathrm{E} U\left(\frac{\theta W+\mathbf{x}}{\theta W+S_{1}}\right)+(1-\lambda) \mathrm{E} U\left(\frac{(1-\theta) W+\mathbf{y}}{(1-\theta) W+S_{2}}\right) \\
& <\mathrm{E} U\left(\lambda \frac{\theta W+\mathbf{x}}{\theta W+S_{1}}+(1-\lambda) \frac{(1-\theta) W+\mathbf{y}}{(1-\theta) W+S_{2}}\right) \\
& =\mathrm{E} U\left(\frac{W+\mathbf{x}+\mathbf{y}}{W+S_{1}+S_{2}}\right)
\end{aligned}
$$

Similarly with the buying price. Define $B_{1}=B(\theta W, \mathbf{x})$ and $B_{2}=B((1-\theta) W, \mathbf{y})$.

$$
\begin{aligned}
1 & =\theta \mathrm{E} U\left(\frac{\theta W+\mathbf{x}-B_{1}}{\theta W}\right)+(1-\theta) \mathrm{E} U\left(\frac{(1-\theta) W+\mathbf{y}-B_{2}}{(1-\theta) W}\right) \\
& <\mathrm{E} U\left(\frac{W+\mathbf{x}+\mathbf{y}-B_{1}-B_{2}}{W}\right)
\end{aligned}
$$

M. Lewandowski

CEJEME 5: 1-34 (2013) 
Risk Attitudes, Buying and Selling...

\subsection{Proof of propositions 5.6 and 5.7}

Notice that for a concave function $f$, the following holds: $f(W+n x)<n f(W+x)$. Using this fact and the definition of a buying price:

$$
\begin{aligned}
& \sum_{i=1}^{m} p_{i} \frac{1}{n}\left[U\left(W+n\left(x_{i}-B(W, \mathbf{x})\right)\right)-U(W)\right] \\
< & \sum_{i=1}^{m} p_{i}\left[U\left(W+x_{i}-B(W, \mathbf{x})\right)-U(W)\right]=0 \\
= & \sum_{i=1}^{m} p_{i} \frac{1}{n}\left[U\left(W+n x_{i}-B(W, n \mathbf{x})\right)-U(W)\right]
\end{aligned}
$$

This implies that $B(W, n \mathbf{x})<n B(W, \mathbf{x})$.

Now we prove proposition 5.7. According to proposition 5.5, for CRRA utility function and given that $n>0$, we have: $S(n W, n \mathbf{x})=n S(W, \mathbf{x})$. And since CRRA functions belong to the class of DARA functions, we know that $S$ is increasing in $W$. Hence $S(W, n \mathbf{x})<S(n W, n \mathbf{x})=n S(W, \mathbf{x})$.

\section{Appendix}

In section 3 two criteria for accepting monetary gamble $\mathbf{x}$ were introduced:

a. wealth from accepting $\mathbf{x}$ should increase on average in nominal terms

b. return from $\mathbf{x}$ should be positive on average

It was claimed that these two criteria are equivalent to the following:

$$
\begin{aligned}
& \text { accept } \mathbf{x} \quad \Longleftrightarrow \quad \mathrm{E}_{\mathrm{a}}(\mathrm{W}+\mathbf{x}) \geq \mathrm{W} \quad \Longleftrightarrow \quad \mathrm{E}_{\mathrm{a}}(\mathbf{x}) \geq 0 \\
& \text { accept } \mathbf{h} \quad \Longleftrightarrow \quad W \times \mathrm{E}_{\mathrm{g}}(\mathbf{h}) \geq \mathrm{W} \Longleftrightarrow \mathrm{E}_{\mathrm{g}}(\mathbf{h}) \geq 1
\end{aligned}
$$

where $\mathbf{h}=1+\frac{\mathbf{x}}{W}$, and $W>L(\mathbf{x})$.

We will explain it on the basis of two simple lotteries. Let $\mathbf{x}=(x, p ; y, 1-p)$ and $\mathbf{h}=(h, q ; k, 1-q)$. Suppose the decision maker with initial wealth $W$ accepts a sequence of $n$ independent gambles of the form $\frac{\mathbf{x}}{n}$. Suppose that $\frac{x}{n}$ occurs $i$ times in the sequence. Then his final wealth will be: $W+\frac{i}{n} x+\frac{n-i}{n} y$. By the law of large numbers as $n$ becomes large, $\frac{i}{n}$ tends to $p$, so that final wealth may be written as:

$$
W+[p x+(1-p) y]=W+\mathrm{E}_{\mathrm{a}} \mathbf{x}
$$

Now suppose that the decision maker with initial wealth $W$ accepts a sequence of $n$ independent multiplicative gambles of the form $(\mathbf{h})^{\frac{1}{n}}$. Suppose that $h^{\frac{1}{n}}$ occurs $i$ 
times in the sequence. Then his final wealth will be: $W h^{\frac{i}{n}} k^{\frac{n-1}{n}}$. By the law of large numbers as $n$ becomes large, $\frac{i}{n}$ tends to $q$, so that final wealth may be written as:

$$
W h^{q} k^{1-q}=W \times \mathrm{E}_{\mathrm{g}} \mathbf{h}
$$

M. Lewandowski 